\title{
Estudios
}

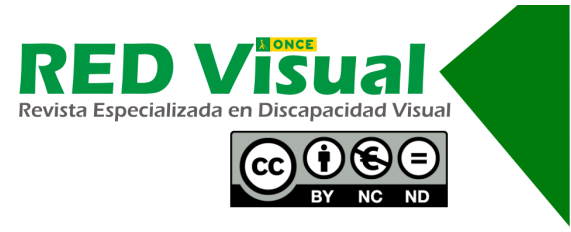

\section{Valoración profesional de la utilidad de la estimulación multisensorial en salas Snoezelen para la atención temprana de diferentes diversidades funcionales, incluida la visual ${ }^{1}$}

\section{Professional assessment of the utility of multi-sensorial stimulation in Snoezelen rooms for early care in the context of visual and other functional diversity}

\author{
A. San José Rubio, I. Asensio Muñoz
}

\section{Resumen}

La estimulación multisensorial constituye una metodología de trabajo necesaria en distintas diversidades funcionales, entre las que se encuentra la visual. En este artículo se presenta una investigación cuyo objetivo es aportar evidencia actualizada acerca de la eficacia y utilidad de las salas Snoezelen de estimulación multisensorial en atención temprana (AT). El estudio empírico se basa en un cuestionario, elaborado ad hoc y validado mediante juicio de expertos, al que responden 28 profesionales que usan estas salas. Los resultados aportan detalles acerca de su efectividad en atención temprana en las diversidades funcionales estudiadas, incluida la visual, útiles como guía para unas buenas prácticas.

\section{Palabras clave}

Discapacidad visual. Estimulación multisensorial. Snoezelen. Atención Temprana. DesarroIlo sensorial y perceptivo.

1 Este artículo es un resumen de un Trabajo Fin de Máster titulado Análisis de la estimulación multisensorial en salas Snoezelen en el ámbito de atención temprana, realizado por Almudena San José y dirigido por Inmaculada Asensio.

San José, A., y Asensio, I. (2020). Valoración profesional de la utilidad de la estimulación multisensorial en salas Snoezelen para la atención temprana de diferentes diversidades funcionales, incluida la visual. RED Visual: Revista Especializada en Discapacidad Visual, 76, 167-189. https://doi.org/10.53094/NMWH4993. 


\begin{abstract}
Multi-sensorial stimulation is a necessary methodology in a number of functional diversities, visual impairment among them. This article introduces research conducted to establish updated evidence of the efficacy and utility of Snoezelen multi-sensorial stimulation rooms (MSR) in early care. The empirical study was based on an ad hoc questionnaire validated by expert opinion, responded to by 28 professionals who use these multi-sensorial rooms. The findings include details on MSR efficacy in early care for the functional diversities studied (visual diversity among them) that may serve as guidelines for good practice.
\end{abstract}

\title{
Key words
}

Visual impairment. Multi-sensory stimulation. Snoezelen. Early care. Sensorial and perceptive development.

\section{Introducción}

El estudio que aquí se presenta se centra en las salas de estimulación multisensorial Snoezelen. Parte de la experiencia de los profesionales como indicador indirecto de su eficacia en el ámbito específico de las edades que delimitan la atención temprana (AT), que abarca de los 0 a los 6 años. La necesidad de este trabajo se justifica, por un lado, por el escaso volumen de investigación existente en nuestro contexto cultural y geográfico, y, por otro, por la poca evidencia empírica acumulada en cuanto a la efectividad de la estimulación sensorial en el ámbito específico de la atención temprana. El estado de la cuestión sobre el tema señala que la mayoría de los estudios proceden del ámbito anglosajón, en el que, en la actualidad, se pueden valorar más verazmente la utilización y eficacia de estos espacios (Gómez, 2009).

De la revisión bibliográfica realizada se deduce, por la cantidad de artículos científicos de los últimos veinte años que cuentan con los términos «estimulación multisensorial» o «Snoezelen» en su título, que el interés por este tema está creciendo notablemente. Las intervenciones multisensoriales, sobre todo en salas Snoezelen, aunque ya tienen un recorrido histórico importante, están de máxima actualidad, lo que se debe, posiblemente, a que, con la mejora y los avances de la tecnología de los últimos tiempos, cada vez son más numerosos y accesibles los recursos disponibles para su utilización en estas salas. La incorporación de los desarrollos tecnológicos está ayudando a una

San José, A., y Asensio, I. (2020). Valoración profesional de la utilidad de la estimulación multisensorial en salas Snoezelen para la atención temprana de diferentes diversidades funcionales, incluida la visual. RED Visual: Revista Especializada en Discapacidad Visual, 76, 167-189. https://doi.org/10.53094/NMWH4993. 
mejor estimulación y está permitiendo, además, que las intervenciones sean más individualizadas, al poder controlar y manipular diferencialmente los materiales. Así, según la necesidad detectada y el tratamiento a realizar, es posible regular la luz, se puede poner música ambiental específica para cada niño, se puede utilizar la vibración en momentos determinados, o se pueden encender o apagar materiales cuando sea oportuno. En definitiva, el avance tecnológico está permitiendo realizar intervenciones cada vez más controladas.

Con respecto a los resultados esperados, en esta investigación se parte de la hipótesis de que los profesionales están totalmente de acuerdo con la intervención en salas multisensoriales (Snoezelen), considerándolo un recurso adecuado para trabajar en los servicios de atención temprana, ya que aportan mayores efectos cuanto más pequeños son los niños. Este presupuesto se apoya en argumentos como los que defienden Etchepareborda, Abad-Mas y Pina-Mulas (2003), cuando afirman que la estimulación multisensorial en un niño pequeño es fundamental para su desarrollo y para sus futuros aprendizajes. Aplicar adecuadamente programas de intervención temprana de manera oportuna permitirá, dentro de las posibilidades de cada niño, acompañarlo en el desarrollo de sus habilidades y capacidades. Y se espera que las áreas del desarrollo más beneficiadas sean las que mayores necesidades planteen en cada niño según su diversidad. Además, se espera que, siguiendo un criterio profesional, se seleccionen y jerarquicen los materiales y la organización del espacio según la utilidad y beneficios percibidos.

\section{Marco teórico}

\subsection{Desarrollo perceptivo}

Para explicar las bases de la estimulación sensorial, partimos de la exposición del proceso general de aprendizaje humano como marco en el que se sitúa el desarrollo perceptivo. Lázaro y Berruezo (2009) elaboran un modelo del proceso de desarrollo, desde la maduración de los sistemas sensoriales hasta el aprendizaje de conductas adaptativas, basándose en el modelo piramidal del aprendizaje realizado por Williams y Shellenberger (1994). Este modelo explica el desarrollo humano representándolo como una pirámide donde la base sobre la que se construyen todos los procesos de aprendizaje es el Sistema Nervioso Central (SNC). Desde esta base, se desarrollan, en orden ascendente, las diferentes fases de desarrollo (Lázaro y Berruezo, 2009), de las

San José, A., y Asensio, I. (2020). Valoración profesional de la utilidad de la estimulación multisensorial en salas Snoezelen para la atención temprana de diferentes diversidades funcionales, incluida la visual. RED Visual: Revista Especializada en Discapacidad Visual, 76, 167-189. https://doi.org/10.53094/NMWH4993. 
cuales las tres primeras se corresponden con el tramo de edad que se asocia con la atención temprana.

La base de la pirámide está constituida por lo que se denominan «sistemas sensoriales básicos» del ser humano, que se corresponden con las estimulaciones básicas del desarrollo táctiles, vestibulares y propioceptivas (Lázaro y Berruezo, 2009). Las vías sensoriales son una estructura importante para el desarrollo del sistema nervioso central, ya que están directamente relacionadas con las vías motrices y con la psique, y es necesario trabajar estos elementos, sobre todo durante la época de mayor plasticidad cerebral, por lo que la estimulación constituye un recurso importante en servicios de atención temprana (Yagüe y Yagüe, 2005).

Por otra parte, en este modelo piramidal se observa la importancia de asimilar los aprendizajes básicos del desarrollo para poder ir consolidando los siguientes. En la educación infantil y en la AT cobra especial relevancia la estimulación sensorial que trabaja con los sistemas sensoriales para asentar los procesos básicos del desarrollo sensorial y poder conseguir los siguientes hitos posteriores para un correcto desarrollo.

Etchepareborda et al. (2003) señalan que, para que un estímulo genere una respuesta en el ser humano, este debe ser adecuado en cantidad y calidad y oportuno en el momento de presentación. Existen tres canales principales para la integración de los estímulos en el cuerpo: el visual, el auditivo y el somatosensitivo.

El uso de ambientes de estimulación multisensorial nace de la importancia que el desarrollo de los sistemas básicos sensoriales tiene, al estar en la base de cualquier tipo de aprendizaje posterior, y de la necesidad de encontrar espacios de observación donde poder aplicar estímulos sobre varios sentidos y reforzar las percepciones sensoriales del mundo que nos rodea (Gómez, 2009), especialmente en niños con dificultades en la integración sensorial o que presentan déficits visuales o auditivos y que requieren, por tanto, un servicio de AT.

\subsection{Estimulación sensorial en atención temprana}

Para Fröhlich (1998), el concepto de estimulación en AT hace referencia a la exposición a diversos estímulos cuya cantidad y duración son determinadas por el terapeuta, lo que produce un proceso de recepción de información a través de los sistemas sensoriales. Él la define como «estimulación basal», porque para procesar

San José, A., y Asensio, I. (2020). Valoración profesional de la utilidad de la estimulación multisensorial en salas Snoezelen para la atención temprana de diferentes diversidades funcionales, incluida la visual. RED Visual: Revista Especializada en Discapacidad Visual, 76, 167-189. https://doi.org/10.53094/NMWH4993. 
los estímulos no se necesita conocimiento o experiencia previa, y porque son la base de la percepción más elevada (Lázaro, Blasco y Lagranja, 2010). Por ello, hace énfasis en la estimulación de las tres áreas básicas de percepción originadas en la etapa embrionaria, que son el área somática, vibratoria y vestibular. Estas tres áreas son la base para el desarrollo óptimo de los sentidos y están estrechamente relacionadas con ellos (Lázaro et al., 2010). A través de la estimulación basal se trabaja también la estimulación sensorial propiamente dicha y, por ello, este concepto se conoce con el nombre de «estimulación multisensorial». En ella, mediante diversos materiales, se estimulan las áreas perceptivas básicas (somática, vibratoria y vestibular), al igual que el resto de sentidos (olfato, gusto, oído, vista y tacto) de manera graduada.

La estimulación multisensorial, para Yagüe y Yagüe (2005), no es una terapia o método complementario, sino una metodología de trabajo imprescindible sobre todo en casos de niños gravemente afectados.

Lázaro et al. (2010) enumeran en su trabajo las competencias que aporta al desarrollo trabajar en un aula multisensorial, que, para ellos, son: la competencia en comunicación lingüística; en el conocimiento e interacción con el mundo físico; la competencia social, la cultural y la artística; la de enseñar a aprender a través de la repetición, y, por último, la competencia en autonomía personal.

\subsection{Sala de estimulación multisensorial (Snoezelen)}

A finales de los años setenta, dos terapeutas holandeses, Jan Hulsegge y Ad Verheul, crearon un espacio sensorial con materiales muy básicos, como tinta mezclada en agua y proyectada en una pantalla, objetos táctiles, instrumentos musicales, un ventilador, botellas de jabón perfumado y distintos alimentos (Gómez, 2009). Este tipo de espacio tuvo mucho éxito en niños con diferentes diversidades funcionales, por ello decidieron crean una sala sensorial más desarrollada y con muchos más recursos, denominándola con el término «Snoezelen», que es la unión de los verbos «oler» y «relajarse» en holandés. Estos terapeutas tuvieron tanta repercusión que en muchos centros se comenzaron a crear los espacios Snoezelen. En 1987, se creó en el Reino Unido la primera sala «Snoezelen» con seis entornos multisensoriales totalmente distintos, y se comprobó que, con el uso de esta sala en pacientes que se autolesionaban, se reducían dichas conductas significativamente (Gómez, 2009). Resultaron tan positivas estas investigaciones, que se comenzaron a crear por casi toda Europa salas multisensoriales para niños con diversidades funcionales, a raíz de

San José, A., y Asensio, I. (2020). Valoración profesional de la utilidad de la estimulación multisensorial en salas Snoezelen para la atención temprana de diferentes diversidades funcionales, incluida la visual. RED Visual: Revista Especializada en Discapacidad Visual, 76, 167-189. https://doi.org/10.53094/NMWH4993. 
la creación de la International Snoezelen Association (ISNA) fundada en 2002 por Ad Verheul y la Dra. Mertens (Lázaro et al., 2010).

Como afirma Cid en el congreso de la ISNA celebrado en Chile en 2012, la estimulación multisensorial se viene realizando desde hace mucho tiempo, y se ha ido perfeccionando con el objetivo de permitir, a personas con algún tipo de alteración sensorial, disfrutar de experiencias sensoriales. Durante los años 90, la práctica de la estimulación multisensorial se difundió a diferentes ámbitos, como el científico y el terapéutico. En España, a partir del año 2000, se comenzó a poner en práctica, y, poco a poco, se van ampliando los servicios de esta terapia y variando sus aplicaciones (Cid, 2012).

Snoezelen es un concepto por el cual se pretenden ofrecer diversos estímulos de manera controlada en un ambiente terapéutico. Para desarrollar una terapia con ese requisito, se requiere una sala de estimulación multisensorial con ciertas características especiales. Snoezelen es la sala ideal, ya que permite controlar los estímulos ofrecidos en cada momento según cada modalidad sensorial. La aplicación de programas de intervención específicos y oportunos permitirá, según las características de cada niño, desarrollar sus habilidades y capacidades (Etchepareborda et al., 2003).

Para Lázaro et al. (2010), los objetivos que se pretenden trabajar en estas salas multisensoriales son: la comunicación oral o mediante sistemas alternativos de comunicación, la interacción con el mundo físico y la autonomía, gracias al uso de la repetición de la estimulación como forma de adquisición de aprendizajes. Además, no cabe duda de que las salas multisensoriales, por su diseño, materiales y la atmósfera de tranquilidad que suscitan, constituyen un espacio perfecto para practicar distintas técnicas de relajación.

\subsubsection{Destinatarios}

En los últimos años, las aulas multisensoriales Snoezelen se están instalando por todo el mundo y en distintos contextos, como hospitales, residencias y centros de día para personas mayores o para personas con diversidad funcional, escuelas, centros infantiles, centros de maternidad y unidades de neonatos, clínicas del dolor, etc. (Cid, 2009).

Lázaro et al. (2010) consideran que ofrecer entornos enriquecidos para niños y niñas en edades de crecimiento, presenten o no alguna diversidad funcional, así como

San José, A., y Asensio, I. (2020). Valoración profesional de la utilidad de la estimulación multisensorial en salas Snoezelen para la atención temprana de diferentes diversidades funcionales, incluida la visual. RED Visual: Revista Especializada en Discapacidad Visual, 76, 167-189. https://doi.org/10.53094/NMWH4993. 
intervenciones educativas adecuadas, constituye una tarea fundamental en las escuelas. Sin embargo, el introducir un área nueva en el currículo de un centro supone un gran esfuerzo de planificación y coordinación con los diferentes profesionales de las escuelas, que tienen que atender a las diferentes pluridiscapacidades. En este sentido, es necesario concretar en mayor grado todos los aspectos curriculares y establecer unos criterios de evaluación adecuados que permitan ver los avances, detenciones o retrasos en el desarrollo.

La práctica en las aulas multisensoriales tiene buenos efectos en niños con diversidad funcional, especialmente para los que presentan diversidad funcional cognitiva y personas con autismo. Además de estas diversidades, se pueden beneficiar también niños con dificultades en el lenguaje, en la percepción sensorial, niños con déficit visual o ceguera, con déficit auditivo o sordera, con diversidad funcional motora -como la parálisis cerebral-, con plurideficiencias - como el caso de las personas con sordoceguera-, etc. Fuera del ámbito educativo, y realizándose de forma más terapéutica, se pueden beneficiar personas con traumatismo craneoencefálico, trastornos de estrés postraumático, dolor crónico y enfermos de alzhéimer (Gómez, 2009).

Según González-Galli (2018), trabajar en un entorno de estimulación sensorial supondrá la obtención de resultados positivos en el tratamiento de diversidades funcionales físicas y psíquicas, en personas que sufren estrés 0 ataques de pánico, en personas con la enfermedad de Alzheimer, con deficiencias visuales, con síndromes tales como el Cri du Chat, niños con trastorno generalizado del desarrollo (TGD), niños con diversidad funcional cognitiva o mental, entre otras.

\subsubsection{Características de las salas multisensoriales}

Las salas multisensoriales son flexibles y no siguen unos patrones estandarizados, ya que van adaptándose según los materiales disponibles, los recursos del centro, las necesidades de cada niño, etc. Es muy importante realizar una valoración del perfil sensorial previo para conocer qué estímulos atraen más al niño y cuáles menos, y de qué manera se puede trabajar con cada niño individualmente según sus características.

Las características que debe poseer una sala multisensorial, descritas por Centenera (2009), son las siguientes: el espacio debe estar insonorizado y apartado de la luz exterior, debe ser accesible para todo tipo de usuarios y estar bien señalizado;

San José, A., y Asensio, I. (2020). Valoración profesional de la utilidad de la estimulación multisensorial en salas Snoezelen para la atención temprana de diferentes diversidades funcionales, incluida la visual. RED Visual: Revista Especializada en Discapacidad Visual, 76, 167-189. https://doi.org/10.53094/NMWH4993. 
además, debe tener en cuenta el espacio de movilidad para poder acceder con sillas de ruedas en su interior o con otros aparatos.

Es fundamental que las dimensiones de la sala permitan crear distintas zonas de estimulación y tener áreas dedicadas especialmente a la estimulación somática, a la estimulación de los sentidos (oído, olfato, gusto y vista) y a la estimulación vestibular. También es importante que el suelo y las paredes estén acolchados, ganando insonorización, y que los elementos que puedan resultar peligrosos, como los enchufes y radiadores, estén protegidos (Campillo, Casanova, Diz y Jaso, 2005; Molina y Banguero, 2008).

Cid (2009), basándose en las condiciones que debe presentar una sala Snoezelen realizadas por Hulsegge y Verheul (1987), menciona que esta sala se debe caracterizar por ser una atmósfera agradable y placentera para los sentidos, con diversas oportunidades para que el niño elija los estímulos. Hay que dedicar el tiempo adecuado al empezar y al acabar las sesiones de estimulación multisensorial, recurriendo a rituales de entrada y salida, y realizar durante la sesión una repetición de la presentación de los estímulos, para que el niño los pueda observar, analizar e integrar. Otro aspecto fundamental es ofrecer en el espacio una amplia oferta de estímulos.

Para la estimulación multisensorial es importante disponer de diversos materiales con los que se trabajen las diferentes áreas de desarrollo. Lázaro y su equipo (2012) consideran fundamental la cama de agua, que consiste, como el nombre indica, en un colchón de agua que incorpora unos altavoces, un calefactor y un inhibidor de olas. ${ }^{2}$ Para Cid (2012), es el aparato principal del espacio Snoezelen, ya que aporta estimulación táctil a la vez que vibratoria, por lo que el área de desarrollo que se ve principalmente favorecida con ella es la perceptiva. Otro material muy usado son las columnas de burbujas (Lázaro, 2012), que son tubos acrílicos que vibran suavemente y que permiten observar la ascensión de las burbujas en su interior, con cambios de color del agua que el niño puede seleccionar. Si se colocan cerca de espejos, favorecen actividades de fijación y seguimiento visual. Además, las columnas que presentan interruptores para variar el color permiten percibir la relación causa-efecto, por lo que estimulan las áreas de desarrollo perceptiva y cognitiva especialmente. El haz de fibra óptica es otro material útil, en el que una fuente luminosa produce una estimulación gracias a que cambia de color lentamente y a que permite realizar movimientos

2 Para poder ver imágenes de estos materiales, se sugiere la consulta de la página web de la Asociación Internacional de Snoezelen: https://snoezelen-professional.com/en/.

San José, A., y Asensio, I. (2020). Valoración profesional de la utilidad de la estimulación multisensorial en salas Snoezelen para la atención temprana de diferentes diversidades funcionales, incluida la visual. RED Visual: Revista Especializada en Discapacidad Visual, 76, 167-189. https://doi.org/10.53094/NMWH4993. 
voluntarios, potenciando así la petición del niño. Con él se desarrolla el área motriz y la perceptiva gracias a que se pueden realizar actividades visuales que estimulan la visión periférica y la agudeza visual (Gómez, 2009). Los focos de colores y proyectores también tienen como objetivo el trabajar la estimulación visual, es decir, el área de desarrollo perceptiva, especialmente en niños con pérdida de campo de visión, a través de actividades como el seguimiento de un foco de luz o discriminar diversos colores. Otro material importante es el panel táctil, visual y auditivo, que consiste en paneles con diversas texturas y estímulos. Gómez (2009) afirma que este tipo de material sirve para trabajar la percepción de sensaciones por medio de la piel, desarrollando el área de desarrollo perceptiva, el área de motricidad fina y el de motricidad gruesa, con su consecuente implicación también en las demás áreas de desarrollo.

Conviene, en este punto, advertir de la importancia que tiene una correcta actitud y formación por parte de los profesionales. Es necesaria la realización de cursos de formación, tanto en protocolos de evaluación como en metodologías específicas de intervención en estas salas, para establecer rituales, provocar respuestas adecuadas, seleccionar qué estímulos se trabajan en la sesión, tomar datos de la evolución de las sesiones y asegurarse de los cambios producidos por el trabajo en estas salas multisensoriales (Lázaro, 2012). La actitud y satisfacción de los profesionales que usan las salas Snoezelen es el punto central en el que se desarrolla la presente investigación.

\subsubsection{Funciones principales de las salas multisensoriales}

La finalidad de este espacio es que los niños puedan estar expuestos a diversos estímulos que les permitan percibir distintas sensaciones y percepciones que ayudarán a mejorar sus procesos de aprendizaje. Huertas (2009) afirma que la sala Snoezelen en una herramienta muy adecuada para trabajar con los niños, ya que estos requieren un aprendizaje por medio de la diversión, el juego y la estimulación. Por ello, es importante que los profesionales usen el juego en sus intervenciones, como reforzador para motivar a los niños a participar en las sesiones de estimulación.

Cid (2012), en el congreso de la ISNA, afirma que las aulas multisensoriales permiten trabajar objetivos individualizados, según las características de cada niño, y, además, favorecen la comunicación y la aparición de conductas adaptadas, disminuyendo los comportamientos disruptivos, reduciendo las estereotipias, favoreciéndole un mayor contacto con el medio externo que le rodea. Kwok y Sung (2003) enumeran algunas funciones que se pueden realizar con la práctica de una intervención en un espacio

San José, A., y Asensio, I. (2020). Valoración profesional de la utilidad de la estimulación multisensorial en salas Snoezelen para la atención temprana de diferentes diversidades funcionales, incluida la visual. RED Visual: Revista Especializada en Discapacidad Visual, 76, 167-189. https://doi.org/10.53094/NMWH4993. 
multisensorial. Entre ellas se encuentran el proporcionar un estado de relajación, desarrollar la autoconfianza del niño, lograr mayor autocontrol de las conductas, fomentar las actividades de exploración y la creatividad de los niños, desarrollar una buena comunicación con el profesional, proporcionar una sensación de bienestar, favorecer la capacidad de elección, mejorar la capacidad de atención y reducir las conductas disruptivas.

Con respecto a las funciones que aporta a nivel fisiológico, Hotz et al. (2006) realizaron un estudio con niños que presentaban lesiones cerebrales, midiéndoles variables fisiológicas como la tasa cardíaca, la concentración de oxígeno en sangre y el tono muscular. Obtuvieron resultados muy positivos con una disminución de la frecuencia cardíaca, una reducción del tono muscular, una reducción de la agitación y un mejor rendimiento cognitivo después de las sesiones en la sala multisensorial (Snoezelen).

En general, según las investigaciones llevadas a cabo por Lázaro (2012), con las intervenciones en las salas multisensoriales se producen mejoras en las conductas de los niños, especialmente en el bienestar emocional y la relajación, además de disminuir la tasa cardíaca. Obtienen que la cama de agua es un elemento fundamental en el trabajo con niños con parálisis cerebral y que consigue disminuir los movimientos estereotipados. Con niños que presentan un trastorno del espectro autista (TEA), observan que los estímulos visuales ayudan a centrar su atención y a relajarse. Consideran que es importante tener en cuenta que hay determinados niños que responden a estímulos, aunque no realicen respuestas observables y afirman que la sala multisensorial se considera un entorno enriquecido.

Para Fowler (2007), la intervención multisensorial promueve la participación activa del niño en la medida de lo posible, permitiendo que pueda aprovechar al máximo las experiencias sensoriales, fomentando su autonomía y la interacción terapeuta-paciente. Este espacio ofrece también la posibilidad de comunicación entre el usuario y el terapeuta mediante los materiales de que dispone la sala multisensorial, por lo que los niños con dificultades de comunicación pueden expresar su estado, sus sentimientos, pensamientos, etc. (Gómez, 2009).

En resumen, el trabajo en las salas multisensoriales conlleva un estado mayor de relajación, mayor bienestar emocional consigo mismo y con las personas que le rodean y una mayor presencia en el mundo (Lázaro et al., 2010).

San José, A., y Asensio, I. (2020). Valoración profesional de la utilidad de la estimulación multisensorial en salas Snoezelen para la atención temprana de diferentes diversidades funcionales, incluida la visual. RED Visual: Revista Especializada en Discapacidad Visual, 76, 167-189. https://doi.org/10.53094/NMWH4993. 


\section{Objetivos}

\subsection{Objetivo general}

El objetivo general de la investigación que se presenta es aportar evidencia acerca de la efectividad y el uso de las salas multisensoriales Snoezelen, a través de la opinión y experiencia de profesionales que trabajan o han trabajado en estos espacios.

\subsection{Objetivos específicos}

- Analizar qué áreas de desarrollo, dentro de la atención temprana, se ven más beneficiadas con la práctica en las salas multisensoriales.

- Identificar qué tipos de discapacidades encuentran mayores efectos positivos con la aplicación de estas salas.

- Comprobar qué edades de los niños en atención temprana se benefician más de las salas.

- Identificar qué espacios, dentro de la sala multisensorial, son más utilizados en la práctica y cuáles tienen mayores efectos según las distintas diversidades funcionales.

- Estudiar qué tipo de materiales son los más utilizados y tienen mayores resultados en la práctica dentro de las salas multisensoriales.

- Comprobar qué tipo de profesional realiza terapia en las salas multisensoriales.

- Identificar la opinión que tienen los profesionales sobre la aplicación de las salas multisensoriales en otros contextos, como la escuela o en el hogar.

\section{Método}

Se opta por una investigación basada en el diseño, validación y aplicación de un cuestionario que tendrá como objetivo medir el uso y la eficacia percibida de las salas multisensoriales (Snoezelen), según la experiencia de los profesionales que las conocen y las han utilizado durante su actividad profesional.

San José, A., y Asensio, I. (2020). Valoración profesional de la utilidad de la estimulación multisensorial en salas Snoezelen para la atención temprana de diferentes diversidades funcionales, incluida la visual. RED Visual: Revista Especializada en Discapacidad Visual, 76, 167-189. https://doi.org/10.53094/NMWH4993. 


\subsection{Instrumento}

Para la construcción del cuestionario, se parte de la información actualizada recogida sobre las salas multisensoriales (Snoezelen) mostrada en el marco teórico, así como de los objetivos propuestos. En la primera parte del cuestionario se plantean una serie de preguntas cerradas, con el objetivo de que los propios profesionales valoren ciertas características de las salas multisensoriales (Snoezelen). La respuesta se ajusta a una escala de tipo Likert de cinco puntos: Totalmente en desacuerdo (1), En desacuerdo (2), Ni de acuerdo, ni en desacuerdo (3), De acuerdo (4), Totalmente de acuerdo (5). En la segunda parte del cuestionario se elaboran una serie de preguntas de elección múltiple, con el objetivo de analizar la frecuencia de la edad y el tipo de diversidad funcional que presentan los niños con los que trabaja la estimulación multisensorial, qué tipo de espacio de la sala multisensorial (Snoezelen) tiene mayor utilidad, qué materiales son los más utilizados en estas salas, qué diversidad funcional dentro de la atención temprana presenta mayores beneficios y que área de desarrollo presenta mejor evolución con la práctica de la estimulación multisensorial (Snoezelen).

Una vez diseñado el cuestionario, se somete a un proceso de validación mediante el método de juicio de expertos, que evalúan cada ítem con una puntuación de 0 a 10 (siendo 0 la peor valoración y 10 la máxima), de acuerdo a tres criterios fundamentales: idoneidad (la pregunta que se formula ¿es una cuestión pertinente de acuerdo a lo que se quiere medir?), relevancia (¿es una pregunta importante?) y claridad (¿se entiende bien?). El comité de expertos que valora se compone fundamentalmente de profesores de la Universidad Complutense de Madrid relacionados con la AT y con el proceso metodológico de elaboración de cuestionarios. Una vez sometido el cuestionario a las valoraciones y opiniones del juicio de expertos, se realizan los cambios y modificaciones oportunas, resultando el cuestionario final que se aplica en la investigación.

Con el cuestionario ya mejorado, se realiza una versión online mediante el uso de la plataforma de Google Formularios. Se elige esta opción con el objetivo de llegar a más muestra. El procedimiento online resulta, por lo general, más cómodo y eficiente. Este modo de presentación es, sin duda, el más accesible, y, una vez completado el cuestionario por los profesionales, las respuestas llegan en el momento y se organizan automáticamente en una base de datos.

San José, A., y Asensio, I. (2020). Valoración profesional de la utilidad de la estimulación multisensorial en salas Snoezelen para la atención temprana de diferentes diversidades funcionales, incluida la visual. RED Visual: Revista Especializada en Discapacidad Visual, 76, 167-189. https://doi.org/10.53094/NMWH4993. 
Después de 4 semanas recogiendo datos, se procede al análisis cuantitativo de los mismos. Para ello, se utiliza la hoja de cálculo Excel y el programa estadístico SPSS. Primeramente, se crea un libro de códigos en SPSS y después se crea la base de datos, añadiendo los datos obtenidos en cada cuestionario. Y, por último, se procede al análisis estadístico de estos datos según el objetivo a lograr, siendo el enfoque más común el descriptivo, a través del cálculo de frecuencias y su representación gráfica, utilizando como herramienta fundamental Excel.

Una vez realizados los análisis estadísticos, se procede a interpretar los resultados y a comprobar si se han logrado los objetivos de la investigación.

\subsection{Muestra y población}

La población que se ha seleccionado para esta investigación está formada por profesionales que tienen experiencia utilizando las salas multisensoriales, esto es, que las han utilizado en algún momento y que conocen su funcionamiento en Madrid.

Para obtener la muestra, se contacta con diferentes centros, tanto educativos como sanitarios, que disponen de una sala o aula multisensorial. En el tiempo fijado se recogen 28 cuestionarios debidamente cumplimentados, de los cuales un $85,7 \%$ proceden de mujeres. Este resultado concuerda con la realidad de una profesión feminizada, en la que el número de mujeres que trabajan en el sector de la salud y educación supera, por lo general, al de varones. La edad de los encuestados va desde los 20 hasta los 40 años, estando el intervalo de mayor participación en las personas con una edad comprendida entre 30 y 40 años.

En cuanto a la profesión del encuestado, podemos comprobar que participan más profesionales del ámbito de la salud, y dentro de este grupo, los profesionales que más participan, con un $28,6 \%$, son los terapeutas ocupacionales; en segundo lugar, los logopedas, y en tercero, los fisioterapeutas. Esta distribución coincide con el perfil más frecuente de especialistas en atención temprana. Por otra parte, estos profesionales tienen experiencia en el uso de las salas multisensoriales con niños que presentan diversidad funcional visual. En la Figura 1 , se pueden observar los demás perfiles profesionales de los participantes del estudio.

San José, A., y Asensio, I. (2020). Valoración profesional de la utilidad de la estimulación multisensorial en salas Snoezelen para la atención temprana de diferentes diversidades funcionales, incluida la visual. RED Visual: Revista Especializada en Discapacidad Visual, 76, 167-189. https://doi.org/10.53094/NMWH4993. 
Figura 1. Perfil profesional de los participantes en el estudio

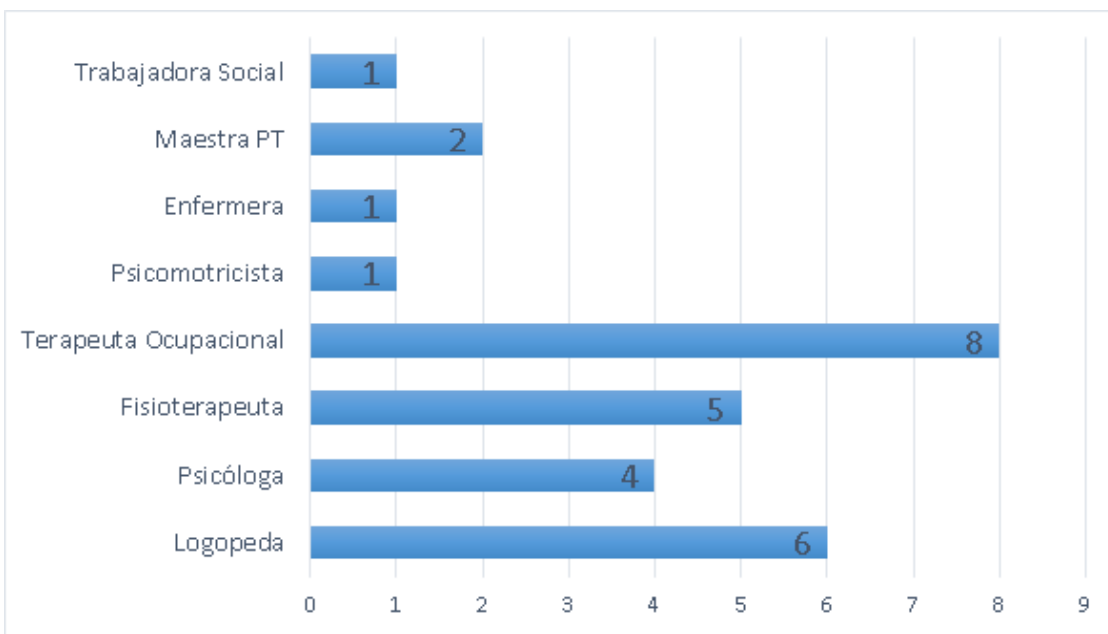

Con respecto a la experiencia que poseen los profesionales encuestados con la práctica profesional en las salas multisensoriales (Snoezelen) (ver Figura 2), encontramos que la mayoría tiene entre menos de 1 año de experiencia y entre más de 6 a 10 años, habiendo solo dos personas que poseen más de 10 años de experiencia en el uso de estas salas. Este dato coincide con lo aportado desde la bibliografía, en la que se indica que hay pocos profesionales que tengan muchos años de experiencia en este ámbito porque la implantación de estas salas se ha ido haciendo de modo paulatino a partir del año 2000.

Figura 2. Años de experiencia profesional en las salas multisensoriales

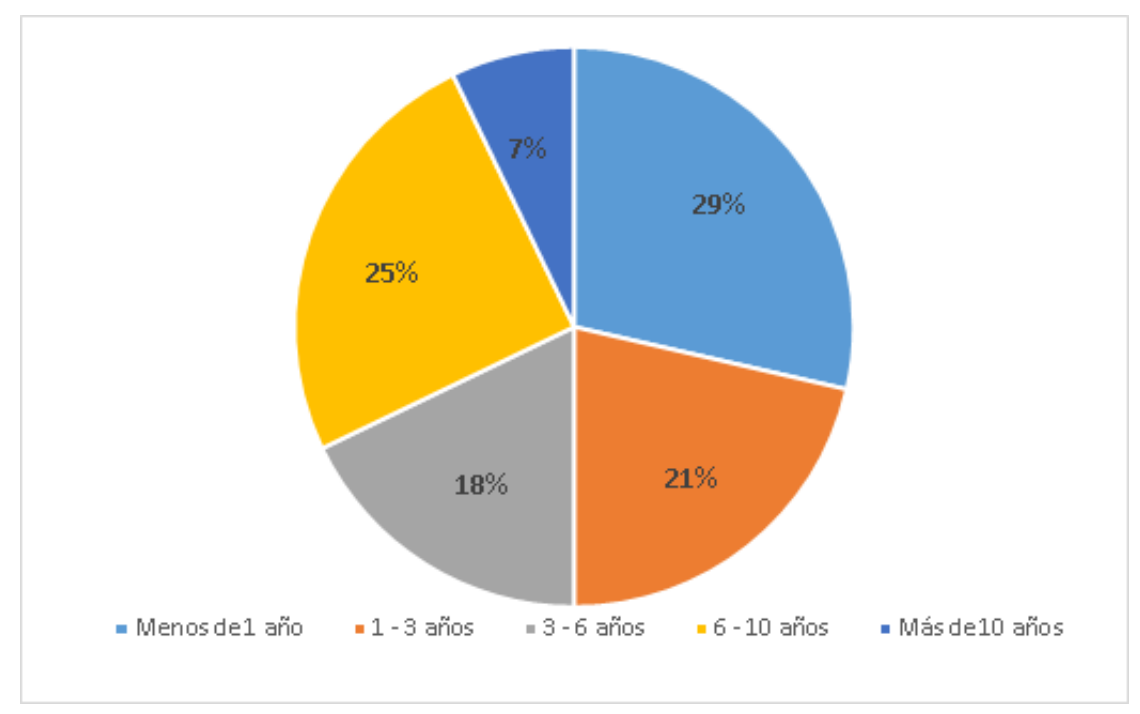

San José, A., y Asensio, I. (2020). Valoración profesional de la utilidad de la estimulación multisensorial en salas Snoezelen para la atención temprana de diferentes diversidades funcionales, incluida la visual. $R E D$ Visual: Revista Especializada en Discapacidad Visual, 76, 167-189. https:/doi.org/10.53094/NMWH4993. 
En cuanto a la formación específica recibida para el uso de salas multisensoriales (Snoezelen), se ha obtenido que un $67,9 \%$ de la muestra ha realizado cursos en algún momento de su carrera profesional para especializarse en la práctica multisensorial. Un $25 \%$ no ha recibido ningún tipo de formación específica en esta práctica.

\section{Resultados}

En primer lugar, se analiza la primera parte del cuestionario, que la constituyen un total de 19 afirmaciones en las que el profesional indica su opinión marcando un número según la escala Likert de cinco puntos definida en el epígrafe anterior. Las afirmaciones con las que están de acuerdo los profesionales, considerando como tales las que presentan una media por encima del 3, son:

- «La práctica en las salas multisensoriales se recomienda como tratamiento complementario a otro».

- «El uso de las salas multisensoriales se puede realizar en otro contexto, como en las escuelas».

- «Estoy satisfecho/a con el trabajo en las salas multisensoriales».

- «Considero que es necesaria una formación específica para intervenir en una sala multisensorial».

- «Es un recurso adecuado para trabajar en servicios de atención temprana».

- «Es una práctica que satisface a las familias».

- «Es una práctica que gusta, especialmente, a los niños».

- «Es una práctica útil».

Analizamos la edad que presentan los niños con los que trabajan en las salas Snoezelen, y se obtiene que un $32,1 \%$ de los profesionales trabajan en las salas multisensoriales con niños que van desde los 2 años hasta mayores de 6 años. Se observa que en niños menores de 2 años no se utiliza con frecuencia esta estimulación multisensorial. 
A continuación, se estudia qué espacio dentro de la sala multisensorial consideran los profesionales que es de mayor utilidad durante la práctica terapéutica, otorgando a cada uno un valor entre 1 y 6 , donde el 1 es el espacio que consideran de mayor utilidad y el 6 el de menor. Partiendo de esto, los valores de la media menores corresponden a los de mayor utilidad y unos valores mayores a los de menor utilidad. En la Tabla 1 se observa que, en los resultados, el espacio que los profesionales consideran de mayor utilidad es el espacio visual, seguido por el auditivo, el táctil y el vestibular y propioceptivo. Por último, como espacios menos valorados se encuentran el espacio olfativo y el gustativo.

Tabla 1. Valoración de la utilidad de los espacios de la sala multisensorial (media y desviación estándar)

\begin{tabular}{|l|c|c|c|c|c|c|}
\hline & $\begin{array}{c}\text { Espacio } \\
\text { visual }\end{array}$ & $\begin{array}{c}\text { Espacio } \\
\text { auditivo }\end{array}$ & $\begin{array}{c}\text { Espacio } \\
\text { táctil }\end{array}$ & $\begin{array}{c}\text { Espacio } \\
\text { olfativo }\end{array}$ & $\begin{array}{c}\text { Espacio } \\
\text { gustativo }\end{array}$ & $\begin{array}{c}\text { Espacio vestibular } \\
\text { y propioceptivo }\end{array}$ \\
\hline N-Válido & 28 & 28 & 28 & 28 & 28 & 28 \\
\hline Media & 2,0000 & 3,0714 & 3,0714 & 4,7143 & 5,0000 & 3,1429 \\
\hline $\begin{array}{l}\text { Desviación } \\
\text { estándar }\end{array}$ & 1,56347 & 0,94000 & 1,01575 & 1,11744 & 1,84592 & 1,55669 \\
\hline
\end{tabular}

Nota: la valoración de la utilidad se realizó mediante una escala de 1 a 6, en la que 1 es el valor máximo de utilidad y 6 el valor menor. De esta forma, a menor resultado en la media, mayor utilidad, y a mayor resultado, menor utilidad.

Centrándonos en la diversidad funcional visual, en la Figura 3 se observa que los profesionales opinan que los espacios que más beneficios aportan a estos niños son el espacio táctil, seguido del visual y del auditivo.

Figura 3. Número de profesionales que valoran útil cada uno de los espacios sensoriales en la intervención con la diversidad funcional visual

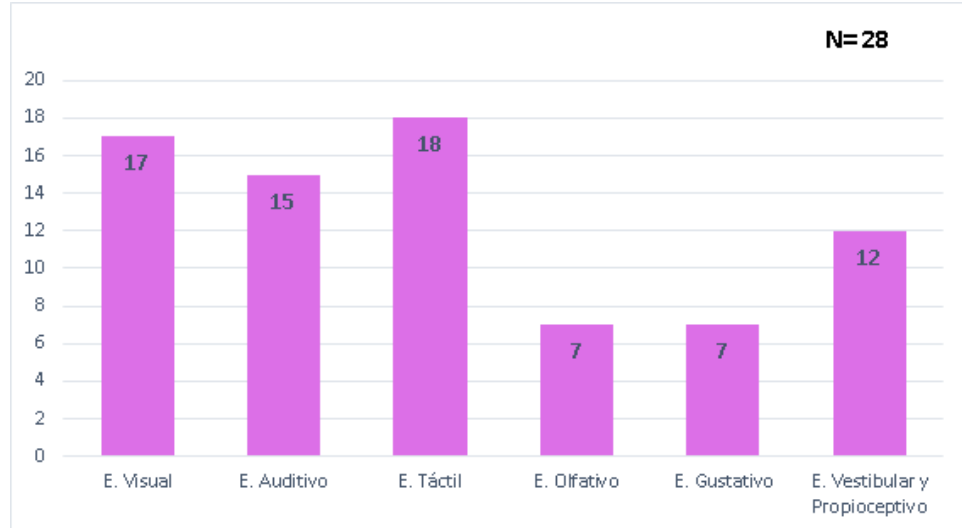

San José, A., y Asensio, I. (2020). Valoración profesional de la utilidad de la estimulación multisensorial en salas Snoezelen para la atención temprana de diferentes diversidades funcionales, incluida la visual. RED Visual: Revista Especializada en Discapacidad Visual, 76, 167-189. https://doi.org/10.53094/NMWH4993. 
En la Figura 4, se presenta el número de profesionales que trabajan con estas salas multisensoriales en cada una de las distintas diversidades funcionales. Observamos que los profesionales trabajan en mayor medida en estas salas Snoezelen con niños con diversidad funcional cognitiva, seguidos en segundo lugar por niños que presentan TEA y después por niños con Parálisis Cerebral y Diversidad Funcional Física.

Figura 4. Número de profesionales que trabajan en cada diversidad funcional con salas multisensoriales

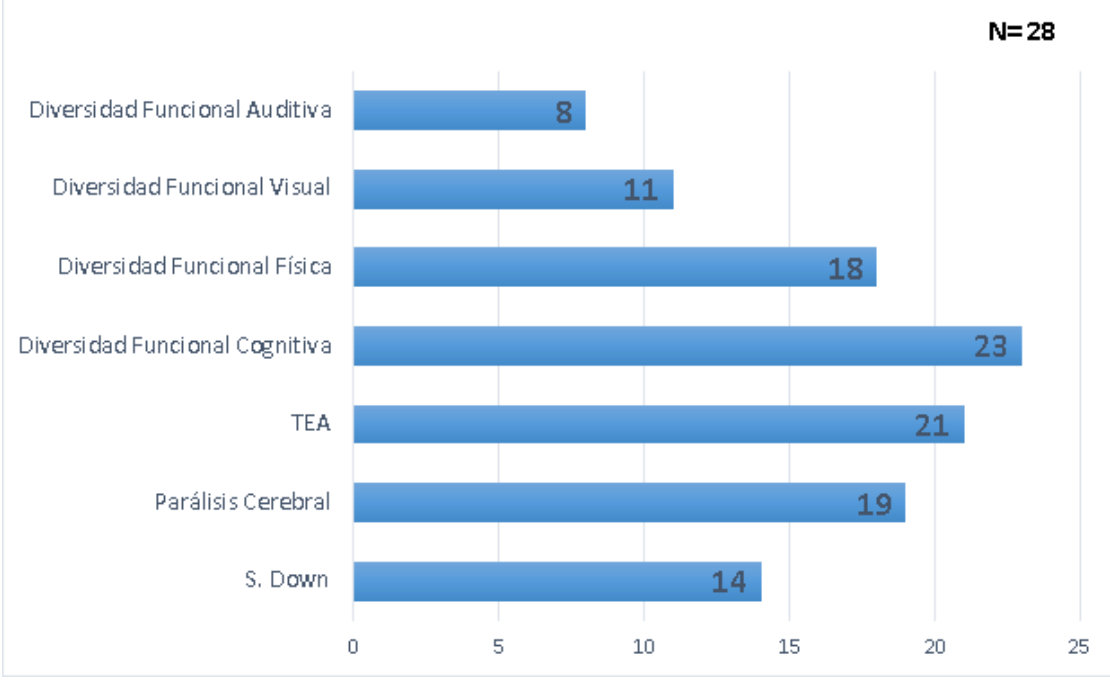

En la Tabla 2 se puede ver qué diversidad funcional consideran los profesionales que obtiene mayor beneficio en el desarrollo a nivel general de los niños con la práctica en las salas multisensoriales (Snoezelen), otorgándole a cada una un valor entre 1 y 6 , donde el 1 significa el mayor beneficio y el 6 el menor.

Tabla 2. Valoración de los beneficios que aportan las salas multisensoriales a cada diversidad funcional (media y desviación estándar)

\begin{tabular}{|l|c|c|c|c|c|c|c|}
\hline & $\begin{array}{c}\text { Síndrome } \\
\text { de Down }\end{array}$ & $\begin{array}{c}\text { Parálisis } \\
\text { Cerebral }\end{array}$ & $\begin{array}{c}\text { Trastorno } \\
\text { Espectro } \\
\text { Autista (TEA) }\end{array}$ & $\begin{array}{c}\text { Diversidad } \\
\text { Funcional } \\
\text { Cognitiva }\end{array}$ & $\begin{array}{c}\text { Diversidad } \\
\text { Funcional } \\
\text { Física / } \\
\text { Motora }\end{array}$ & $\begin{array}{c}\text { Diversidad } \\
\text { Funcional } \\
\text { Visual }\end{array}$ & $\begin{array}{c}\text { Diversidad } \\
\text { Funcional } \\
\text { Auditiva }\end{array}$ \\
\hline N-Válido & 28 & 28 & 28 & 28 & 28 & 28 & 28 \\
\hline Media & 3,9643 & 3,2143 & 3,2857 & 4,2857 & 4,2857 & 3,9286 & 5,0357 \\
\hline $\begin{array}{l}\text { Desviación } \\
\text { estándar }\end{array}$ & 1,95282 & 2,13189 & 2,38603 & 1,88281 & 1,80241 & 1,41234 & 1,93376 \\
\hline
\end{tabular}

Nota: la valoración de la utilidad se realizó mediante una escala de 1 a 6 , en la que 1 es el valor máximo de utilidad y 6 el valor menor. De esta forma, a menor resultado en la media, mayor utilidad, y a mayor resultado, menor utilidad.

San José, A., y Asensio, I. (2020). Valoración profesional de la utilidad de la estimulación multisensorial en salas Snoezelen para la atención temprana de diferentes diversidades funcionales, incluida la visual. $R E D$ Visual: Revista Especializada en Discapacidad Visual, 76, 167-189. https://doi.org/10.53094/NMWH4993. 
Observamos, en primer lugar, que la diversidad funcional que los profesionales consideran que recibe mayores beneficios, en su desarrollo general, gracias a la práctica multisensorial, es la Parálisis Cerebral, seguida, con muy poca diferencia, del Trastorno Espectro Autista (TEA) y, en segundo lugar, se encontrarían la Diversidad Funcional Visual y el síndrome de Down. Por último, las diversidades que según los profesionales obtienen menos beneficios son la Diversidad Funcional Cognitiva, la Diversidad Funcional Física y la Diversidad Funcional Auditiva.

A continuación, se analiza el tipo de materiales de que disponen las salas multisensoriales donde trabajan los profesionales encuestados. En la Figura 5 podemos observar que los materiales más utilizados en las salas Snoezelen son la música ambiental, el tubo de burbujas, la cama de agua y el panel táctil y visual. Los espejos, focos, proyectores y pulsadores se encuentran también entre los materiales que han obtenido mayor frecuencia de elección.

Figura 5. Número de profesionales que utilizan cada uno de los materiales de la sala multisensorial

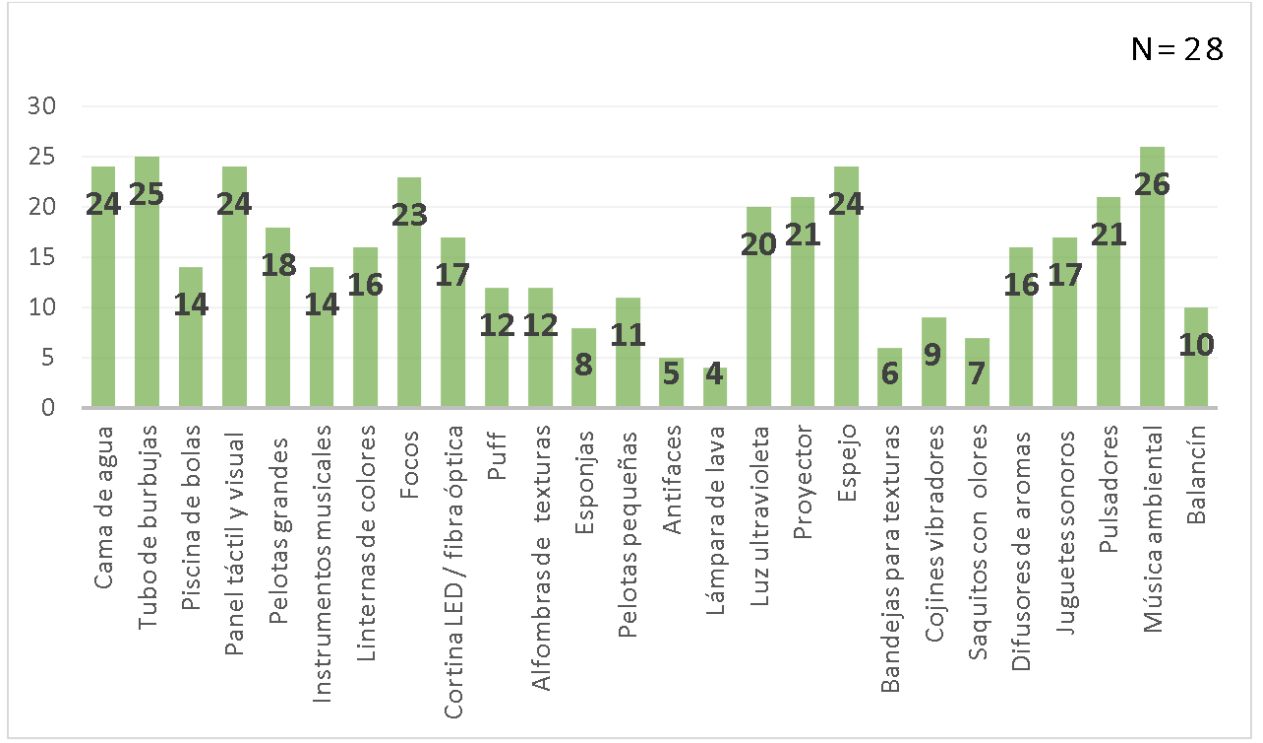

Por último, se obtiene información sobre las áreas de desarrollo que presentan más beneficios para cada una de las diferentes diversidades funcionales. Según la perspectiva de los profesionales consultados, el área perceptiva se coloca en primer lugar en la mayoría de las distintas capacidades que se estudian. En segundo lugar, se sitúa el área comunicativa, presentando buenas puntuaciones en las diferentes capacidades, especialmente en la diversidad funcional cognitiva, en el TEA y en las 
diversidades funcionales visuales y auditivas. Otra de las áreas del desarrollo que obtiene buenos beneficios es el área cognitiva, favoreciendo principalmente a niños con síndrome de Down, con diversidad funcional cognitiva y con diversidad funcional visual. La siguiente área del desarrollo es el área motora gruesa, beneficiando especialmente a niños con Parálisis Cerebral y con Diversidad Funcional Física.

Centrándonos específicamente en la Diversidad Funcional Visual, en la Figura 6 se puede observar que los profesionales consideran que las áreas del desarrollo que presentan mejores resultados con la práctica multisensorial en salas Snoezelen son el área perceptiva, seguida del área comunicativa principalmente, y de las áreas cognitiva, social y de autonomía.

Figura 6. Número de profesionales que indican en cada área que esta se beneficia con el uso de las salas multisensoriales

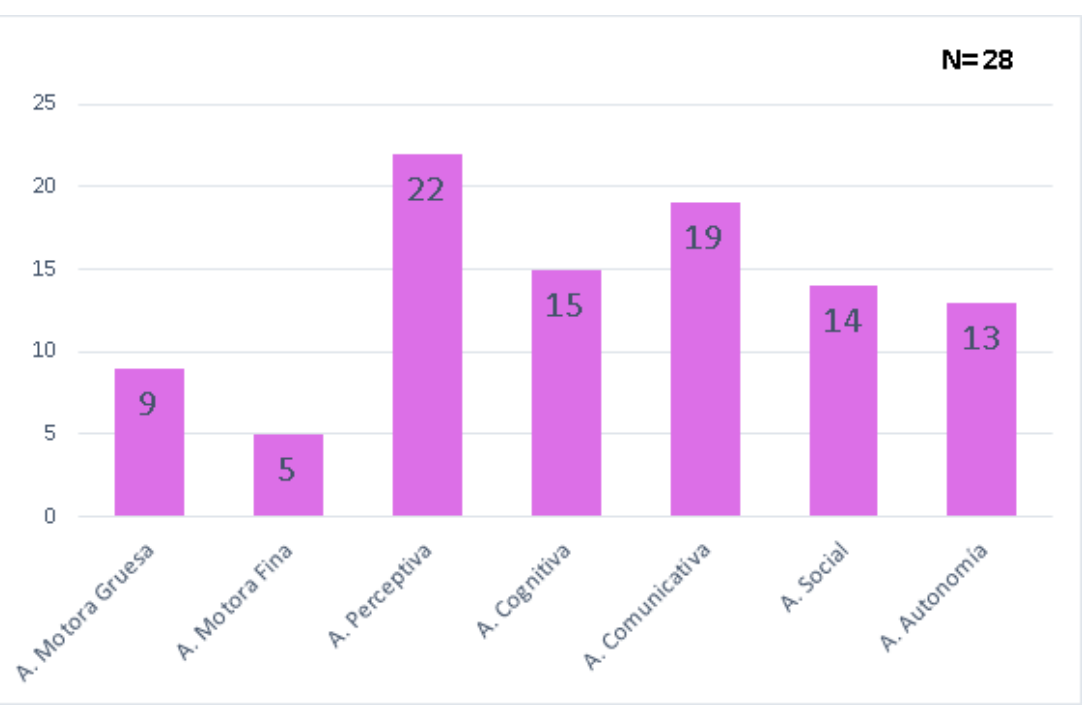

Estos resultados indican que, de manera general, las áreas del desarrollo que presentan mayores beneficios son aquellas en que se observan mayores necesidades según la diversidad funcional que se trabaja.

\section{Discusión y conclusiones}

En primer lugar, la conclusión a la que se llega, tras la revisión de fuentes y el análisis de los cuestionarios aplicados a diferentes profesionales que han trabajado con estas 
salas, es que tanto los profesionales encuestados como diferentes autores de estudios acerca las salas Snoezelen consideran que se producen cambios en diferentes áreas del desarrollo para niños con diversidades funcionales, y que los niños, al trabajar en estas salas, se muestran muy receptivos, con una mayor activación y participación, lo que supone un proceso de avance en sus aprendizajes.

Los profesionales están de acuerdo en que el uso de las salas Snoezelen es útil en otros contextos, más allá de la atención temprana, como pueden ser las escuelas. Numerosos autores están a favor de implementar estas salas dentro de colegios como aulas multisensoriales, sobre todo en colegios de educación especial. Lázaro et al. (2010) indican que ofrecer entornos enriquecidos multisensoriales para niños en edades de crecimiento, presenten o no alguna diversidad funcional, constituye una tarea fundamental en las escuelas. Con respecto a si se puede dar un uso en el contexto del hogar, los profesionales no lo consideran tan apropiado, ya que las salas no tendrían la misma función y servirían, simplemente, como un espacio para relajar al niño.

Por último, como principal aportación del estudio aquí realizado, cabe destacar que este trabajo es útil en el campo de la atención temprana como guía para el desarrollo de la práctica de las salas Snoezelen. Se aporta información sobre los beneficios que se obtienen con ellas de manera coincidente con lo señalado por la bibliografía consultada, pero ajustada, al mismo tiempo, a las condiciones de trabajo real de los especialistas en atención temprana de nuestro entorno más inmediato y en nuestro contexto geográfico. El trabajo también deja claro que la estimulación multisensorial es una práctica que, a juzgar por los especialistas, produce mejoras en diferentes áreas del desarrollo para niños con diversidades funcionales, lo que supone un progreso en sus aprendizajes. Las áreas del desarrollo que presentan mejores resultados en diversidad funcional visual son la perceptiva y la comunicativa, y la que menos el área motora fina.

Este estudio se basa fundamentalmente en la opinión de los expertos, pero, para la obtención de evidencias, como prospectiva de investigación, habría que llevar a cabo estudios que permitieran, en cada caso, analizar el efecto de intervenciones multisensoriales específicas para las distintas diversidades funcionales, con el fin de determinar si la intervención provoca mejoras y si estas son perdurables en el tiempo. Sería conveniente el uso de diseños pre y post con grupo de control, de modo que se pueda valorar si el tratamiento multisensorial produce beneficios con una escala o

San José, A., y Asensio, I. (2020). Valoración profesional de la utilidad de la estimulación multisensorial en salas Snoezelen para la atención temprana de diferentes diversidades funcionales, incluida la visual. RED Visual: Revista Especializada en Discapacidad Visual, 76, 167-189. https://doi.org/10.53094/NMWH4993. 
inventario de desarrollo que cuantifique resultados en las distintas áreas, para obtener datos más objetivos y fiables. Como afirman Sanz et al. (2004), la efectividad de la estimulación sensorial todavía no está suficientemente demostrada científicamente, ya que, en la mayoría de casos, los estudios realizados cuentan con un número reducido de pacientes y no poseen un grupo control. Coincidimos con la Asociación Internacional de Snoezelen (ISNA), que informa de que hay unanimidad en la necesidad de más y mejor experimentación para poder aportar evidencia científica sobre el efecto de la intervención en un espacio Snoezelen en las personas. Pero una aproximación como la que aquí se presenta aporta una panorámica general que puede resultar útil como guía para la práctica.

\section{Referencias bibliográficas}

Campillo, M. J., Casanova, I. M., Diz, M. E., y Jaso, M. (2005). Actuación del fisioterapeuta en la terapia de estimulación multisensorial en niños con necesidades educativas especiales. Fisioterapia, 27(3), 161-166. (DOI: https://doi.org/10.1016/S0211-5638(05)73432-5).

Centenera, M. (2009). Estimulación neurosensorial en alumnos especialmente afectados [PDF]. Psicología Educativa, 15(1), 21-25.

Cid, M. J. (2009). Estimulació Multisensorial en un espai Snoezelen en persones adultes amb greu discapacitat intel/lectual [PDF]. Tesis doctoral. Tarragona, España: Universidad Rovira i Virgili.

Cid, M.J. (2012). Estimulación multisensorial: espacios Snoezelen [PDF]. Ponencia presentada en la I Jornada Chilena sobre Estimulación Multisensorial, 24 de octubre de 2012, San Francisco de Mostázal, Chile.

Etchepareborda, M. C., Abad-Mas, L., y Pina-Mulas, J. (2003). Estimulación multisensorial. Revista de Neurología, 36(1), 122-128. (DOI: https://doi.org/10.33588/rn.36S1.2003004).

Fowler, S. (2007). Sensory stimulation: sensory-focused activities for people with physical and multiple disabilities. Londres, Reino Unido: Jessica Kingsley.

Fröhlich, A.D. (1998). Basale Stimulation: ein Konzept für die Arbeit mit schwer beeinträchtigten Menschen. Düsseldorf, Alemania: Verlag Selbstbestimmtes Leben.

San José, A., y Asensio, I. (2020). Valoración profesional de la utilidad de la estimulación multisensorial en salas Snoezelen para la atención temprana de diferentes diversidades funcionales, incluida la visual. RED Visual: Revista Especializada en Discapacidad Visual, 76, 167-189. https://doi.org/10.53094/NMWH4993. 
Gómez, M.C. (2009). Aulas multisensoriales en educación especial: estimulación e integración sensorial en los espacios snoezelen. Vigo, España: Ideaspropias.

González-Galli, A.M. (2018). Salas de estimulación multisensorial [página web]. ISNAmse.org.

Hotz, G.A., Castelblanco, A., Lara, I. M., Weiss, A.D., Duncan, R., y Kuluz, J.W. (2006). Snoezelen: a controlled multi-sensory stimulation therapy for children recovering from severe brain injury [PDF]. Brain Injury, 20(8), 879-888. (DOI: https://doi.org/ 10.1080/02699050600832635).

Huertas, E. (2009) La sala Snoezelen en terapia ocupacional: a propósito de un caso de daño cerebral infantil [PDF]. TOG, 6(10), 1-7.

Hulsegge, J., y Verheul, A. (1987). Snoezelen: another world: a practical book of sensory experience environments for the mentally handicapped. Chesterfield, Reino Unido: Rompa.

Kwok Y.F., y Sung H.F. (2003). The application of a multisensory Snoezelen room for people with learning disabilities - Hong Kong experience [PDF]. Hong Kong Medical Journal, 9(2), 122-126.

Lázaro, A. (2012). La práctica educativa en aulas multisensoriales: Atmósferas para el crecimiento y el desarrollo humanos. Zaragoza, España: Mira.

Lázaro, A., y Berruezo, P. (2009). La pirámide del desarrollo humano [PDF]. Revista Iberoamericana de Psicomotricidad y Técnicas Corporales, 34(9), 15-42.

Lázaro, A., Blasco, S., y Lagranja, A. (2010). La integración sensorial en el aula multisensorial y de relajación: estudio de dos casos [PDF]. Revista Electrónica Interuniversitaria de Formación del Profesorado, 13(4), 321-334.

Molina, T., y Banguero, L. F. (2008). Diseño de un espacio sensorial para la estimulación temprana de niños con multidéficit [PDF]. Revista Ingeniería Biomédica, 2(3), 40-47.

Sanz, S., De Pobes, A., Bové, M.P., Tàsies, S., Andrés, B., Noguera, A. M., Soriano, M., y Roig, M. T. (2004). Terapia ocupacional en el estado vegetativo y de mínima conciencia: estimulación sensorial [PDF]. Mapfre Medicina, 15(2), 112-117.

San José, A., y Asensio, I. (2020). Valoración profesional de la utilidad de la estimulación multisensorial en salas Snoezelen para la atención temprana de diferentes diversidades funcionales, incluida la visual. RED Visual: Revista Especializada en Discapacidad Visual, 76, 167-189. https://doi.org/10.53094/NMWH4993. 
Williams, M. S., y Shellenberger, S. (1994). The Alert Program for self-regulation. American Occupational Therapy Association. Sensory Integration. Special Interest Section Newsletter, 17, 1-3.

Yagüe, M.P., y Yagüe, M.M. (2005). Estimulación multisensorial en el trabajo del fisioterapeuta pediátrico. Fisioterapia, 27(4), 228-238. (DOI: https://doi.org/10.1016/ S0211-5638(05)73443-X).

Almudena San José Rubio. Logopeda. Estudiante del Máster de Atención Temprana. Universidad Complutense de Madrid. Facultad de Educación-Centro de Formación del Profesorado. Calle Rector Royo Villanova, s/n; 28040 Madrid (España). Correo electrónico: almudena.sanjose@hotmail.com.

Inmaculada Asensio Muñoz. Profesora titular. Departamento de Investigación y Psicología en Educación. Universidad Complutense de Madrid. Facultad de Educación-Centro de Formación del Profesorado. Calle Rector Royo Villanova, s/n; 28040 Madrid (España). Correo electrónico: macu@edu.ucm.es. 